\title{
A New Behavioural Assessment Tool for Patients with Major Neurocognitve Disorders: Results of a Clinical Study
}

\section{Atul Sunny Luthra*}

Homewood Health Centre, Canada

\begin{abstract}
120 residents with dementia in long term care facilities were recruited for the study. Pittsburg Agitation Scale was used to screen for the presence of BPSD. 60 patients were in the control group and 60 patients in the study group. Construct and criteria validity was established by comparing LuBAIR to BEHAVE-AD and Cohen-Mansfield Agitation Inventory. Intra- and inter-rater reliability of LuBAIR was also established. A Clinical Utility Survey (CUS) survey was developed for the study to determine the usefulness of LuBAIR on three variables: less labor intensive, more comprehensive and better categorization of behaviors in clinical meaningful categories. Intra-rater reliability was established for 8 of 12 and inter-rater reliability for 10 of 12 behavioral categories. LuBAIR had comparable Construct and Criteria Validity. CUS findings showed $23 \%$ of nurses found LuBAIR less labor intensive, $77 \%$ found it more comprehensive and $98 \%$ agreed LuBAIR helps understand behaviors in clinically meaningful ways.
\end{abstract}

Keywords: Agitation; LuBAIR; Intervention response; Psychological symptoms

\section{Introduction}

To alleviate stress for the patient and care providers, expanding knowledge and creating evidence-based pharmacological [1-3] and non-pharmacological $[4,5]$ treatments for Behavioral and Psychological Symptoms of Dementia (BPSD) has been identified to be a top priority for Dementia/Major Neurocognitive Disorders (D/NCD) research (equivalent to "behavioral symptoms of neurocognitive disorder" in DSM-5) [6,7].

Agitation, as a symptom, can be the presentation of a wide variety of clinical syndromes/states in patients with D/NCD [8]. As an example, in an acute confused state of delirium, agitation can be present in conjunction with disorganized thinking, delusions, hallucinations, emotional dysregulation and circadian rhythm disturbances. In an acute psychotic state, agitation may be present alongside thought content and form abnormalities, as well as perceptual abnormalities. Similarly, a pathological emotional state of severe clinical depression can present as agitation, mood congruent content abnormalities, emotional depravity and circadian rhythm dysregulation. Unmet physiological needs in the form of severe pain, urinary retention or fecal impaction can show itself as agitation, emotional dysregulation or circadian rhythm disturbance. Any of the aforementioned clinical syndromes can be present in patients with varying stages of D/NCD; early, middle or late stages.

In the early stages of $\mathrm{D} / \mathrm{NCD}$, when it is possible to obtain a reasonably reliable history and conduct a formal mental state examination with an appropriate physical examination, diagnosis of individual psychiatric syndromes using established DSM-5 criteria can be successfully achieved. The same can be achieved with the application of Cohen-Mansfield criteria to diagnose agitation in patients with early stages of D/NCD [9].

With the advancement of D/NCD into the moderate to advanced stages, there is decreased reliability and validity of the history and mental status examination conducted with the patient. While assessing non-cognitive symptoms in moderate to advanced stages of D/NCD, greater emphasis is put upon gathering collateral information for varied sources and on direct clinical observations; more so than direct patient interview. Under such circumstances, diagnosing specific clinical syndromes/states or distinguishing amongst them using DSM criteria is increasingly difficult and with decreasing validity and reliability.
Standardized scales are used in clinical practice to distinguish amongst aforementioned clinical syndromes/states, albeit not BPSD per se, in patients with D/NCD. This step is both a prerequisite and essential, as the diagnosis of BPSD in accordance with DSM criteria is one of exclusion. All major mood, anxiety and psychotic disorders, delirium, unmet physiological needs, medical and milieu contributors all have to be ruled out in order to diagnose BPSD [10].

Example of the most commonly used standardized scale to identify mood disorder in D/NCD in clinical practice is Cornell Scale for Depression [11]. Another, less commonly used scale is the Dementia Mood Assessment Scale [12]. These two scales are commonly used in clinical practice to assess depression in patients with $\mathrm{D} / \mathrm{NCD}$. The primary use of these two scales is to measure the severity of the depression, but not to screen for the presence of depression. The diagnosis of depression has to be made through other clinical means prior to administration of these scales to quantify the severity of the depression. Even in the original article by [12], the author states "The Cornell Scale is a quantitative measure of depression. Although its total scores correlate with the presence of depressive syndromes classified by research diagnostic criteria (RDC), Cornell Scale is not designed for use as a diagnostic instrument." Examples of scales used to detect psychotic symptoms in patients with D/NCD include Neuropsychiatric Inventory and Behavioral Pathology in Alzheimer Disease Rating Scale (BEHAVE-AD) $[13,14]$. These two scales are also used to detect anxiety and psychotic symptoms in the same cohort of patients. However, during advanced stages of $\mathrm{D} / \mathrm{NCD}$, when clinical examination becomes unreliable and the incidence of BPSD increases, the reliability of these scales in distinguishing a clinical state from a clinical syndrome is unknown $[13,14]$.

The most commonly used scale to screen for delirium in clinical practice is Confusion Assessment Methodology (CAM) [15]. CAM

*Corresponding author: Atul Sunny Luthra, Homewood Health Centre, Canada, Tel: 2265001893; E-mail: SLuthra@homewoodhealth.com

Received October 12, 2015; Accepted December 21, 2015; Published December 28, 2015

Citation: Luthra AS (2015) A New Behavioural Assessment Tool for Patients with Major Neurocognitve Disorders: Results of a Clinical Study. J Gerontol Geriatr Res 4: 260 . doi:10.4172/2167-7182.1000260

Copyright: (C) 2015 Luthra AS. This is an open-access article distributed under the terms of the Creative Commons Attribution License, which permits unrestricted use, distribution, and reproduction in any medium, provided the original author and source are credited. 
Citation: Luthra AS (2015) A New Behavioural Assessment Tool for Patients with Major Neurocognitve Disorders: Results of a Clinical Study. J Gerontol Geriatr Res 4: 260. doi:10.4172/2167-7182.1000260

Page 2 of 9

has been primarily validated in screening of delirium in hospitalized, non-cognitively impaired, older adults [15]. CAM has recently been validated for clinical use to screen for delirium in older adults with mild D/NCD [16]. However, the reliability and validity of CAM to screen for delirium in moderate to advances stages of $\mathrm{D} / \mathrm{NCD}$ is yet to be determined.

In accordance with DSM-5, the diagnosis of BPSD is one of exclusion. All the major mood, anxiety and psychotic disorders, delirium, dysregulation in innate physiological needs and milieu contributors have to be ruled out before a diagnosis of BPSD can be made. Even with application of all the aforementioned scales in patients with moderate to advanced $\mathrm{D} / \mathrm{NCD}$, it remains a challenge to not only diagnose individual clinical syndromes, but also to distinguish amongst these syndromes [13]. As a result, the diagnosis of BPSD in patients with moderate to advanced stages of $\mathrm{D} / \mathrm{NCD}$ lacks reliability and validity. Yet, it is in the moderate to advanced patient population of $\mathrm{D} / \mathrm{NCD}$ that the prevalence of BPSD reaches as high as $90 \%$.

According to all recent literature, one way forward is to move away from the focus of labelling and towards understanding the meaning for the presence of behaviors in patients with $\mathrm{D} / \mathrm{NCD}[16,17]$. Understanding the meaning of behaviors is being proposed as an essential step in order to make substantive progress in the pharmacological and behavioral intervention of behaviors in $\mathrm{D} / \mathrm{NCD}[18]$.

The first necessary step in achieving this aforementioned goal was to propose a comprehensive biopsychosocial (BPS) model for occurrence of behaviors in patients with D/NCD. All existing models are dichotomized along Biological and Psychosocial [19] paradigms. Literature has identified biological (stage of the disease, inherent circadian rhythms, innate physiological needs), Personal factors (pre-morbid personality, acquired coping mechanisms), and Environmental factors (milieu structure, interpersonal interactions) as being contributory to the occurrence of behaviors in patients with $\mathrm{D} /$ NCD. A new model was posited which incorporated all the identified biopsychosocial (BPS) variables in the generation of behaviors in patients with $\mathrm{D} / \mathrm{NCD}$. The new proposed terminology to accurately reflect the BPS basis for the presence of behaviors in D/NCD is titled; Stage Congruent Responsive Behaviors (SCRB; pronounced 'scrub') [20]. Subsequently, direction was sought from published literature to identify the most appropriate approach to classify this newly appointed terminology; SCRB [19].

Criteria put forth by Davis [21] were identified as the most appropriate way to develop a reliable and valid measure of classification for behaviors in $\mathrm{D} / \mathrm{NCD}$. These criteria were:

1. Identification of the target population (D/NCD),

2. Construction of items into clinically meaningful categories which adequately represent the domain,

3. Definition of the purpose of the measure or meaning of each behavioral category, and

4. Identification of a Specification of the Construct for each domain or clinically meaningful category.

\section{Identification of Target Population}

The target population includes D/NCD patients with moderate to advanced stages of the disease.

\section{Construction of items into categories}

This step involved recognition of individual behavioral symptoms and clustering them into discrete and individual categories. Each of these clusters of symptoms represents a "clinically meaningful" category of behaviors in patients with $\mathrm{D} / \mathrm{NCD}$.

\section{Definition of the purpose of the measure}

Each clinical category identified represents a specific purpose or meaning being served for the patient with $\mathrm{D} / \mathrm{NCD}$.

\section{'Specification of the construct' for each category}

Specification of Theoretical Constructs (STC) was identified from established behavioral and developmental psychology literature to justify the formation of each individual, clinically meaningful behavioral category. Varied STC identified to justify the formation of individual behavioral categories were [20]:

- Behaviors based in information processing theories

- Behaviors based in motivational and needs-based theories

- Behaviors based in theories on regulation of emotions

- Behaviors based in theories on principles of compliance and aggression

- Heterogeneous group which encompasses each of the above STC to account for the varied subtypes of behaviors in this category.

Behavioral categories represented under each of the above constructs are:

\section{Behaviors based in information processing theory}

1. Disorganized Behaviors

2. Misidentification Behaviors

Behaviors based in needs based and motivational theories

1. Apathy Behaviors

2. Goal Directed Behaviors

3. Motor Behaviors

4. Importuning Behaviors

\section{Behaviors based in theories on regulation of emotions}
1. Emotional Behaviors
2. Fretful/Trepidated Behaviors

Behaviors based in theories on principles of compliance and aggression

1. Oppositional Behaviors

2. Physically Aggressive Behaviors

\section{Heterogeneous group}

\section{Vocal Behaviors \\ 2. Sexual Behaviors}

Each of these individual, clinically meaningful, behavioral categories was used to develop a new behavioral measurement tool titled Luthra's Behavioral Assessment and Intervention Response (LuBAIR) Scale (see Appendix 1). A clinical study to establish the reliability and validity of this newly developed scale was submitted to Research Ethics Board, 
McMaster University, Hamilton, Canada. The study was approved in 2009.

\section{Objectives}

Primary objective of this study was to establish the reliability and validity of a new behavioral assessment scale to measure BPSD; Luthra's Behavioral Assessment and Intervention Response (LuBAIR) Scale in D/NCD. It was hypothesized that LuBAIR;

1. Has equivalent face, content and criteria validity in comparison to two existing behavioral scales (Cohen-Mansfield Agitation Inventory-CMAI) and Behavioral Pathology Scale in Alzheimer's Disease-BEHAVE-AD),

2. Has acceptable intra- and inter-rater reliability in comparison to CMAI and BEHAVE-AD,

3. Is less labor-intensive to score when compared to the above two behavioral assessment scales,

4. Is more comprehensive in scope when compared to existing behavioral scales, and

5. Is better able to categorize behaviors into clinically meaningful categories.

\section{Methods}

The following section describes the study population, sample size, measures, participant recruitment, study design, methodology, hypothesis, and definition of endpoints.

\section{Study population}

The population for this study consisted of residents living in longterm care facilities previously diagnosed with D/NCD. Seven (7) long term care facilities (LTCF) were approached to participate in the study. Five (5) of the LTCF were in the City of Hamilton, Ontario, Canada and two (2) of the LTCF were in the City of Burlington, Ontario, Canada.

\section{Inclusion criteria}

1. Have an existing diagnosis of moderate to advanced D/NCD as determined by the resident's physician. The diagnosis of $\mathrm{D} / \mathrm{NCD}$ was confirmed by a research assistant (RA) using the Mini Mental State Examination (MMSE). A score of twenty-three (23) or less supports the diagnosis $[16,22,23]$.

2. Residents were either capable of consenting to participate in the study or had a Power of Attorney (POA) or Substitute Decision Maker (SDM) who was able and willing to consent on behalf of the resident.

3. Resident or their POA/SDM was able to read, comprehend and speak in English at a minimum of level of grade six (6). The 'letter of information' for the SDM was written at a grade six (6) level of comprehension.

4. Residents were screened for the presence of BPSD using Pittsburg Agitation Inventory (PAI). Residents with a score of three (3) or higher were included in the study group. Residents with a score of two (2) or less on PAI were included in the control group [24].

\section{Exclusion criteria}

1. Residents who had in their care plan a transfer to another LTCF during the study time period.
2. Residents who were not given a diagnosis of $\mathrm{D} / \mathrm{NCD}$ by their physician or residents who were given a diagnosis of D/NCD but who scored more than twenty-three (23) on Mini Mental State Examination (MMSE).

3. Residents or their POA/SDM were unable to understand, read or speak English and were unable to complete the study assessment tools.

4. Residents or their POA/SDM were unable or unwilling to provide consent to participate in the study.

\section{Sample size}

A total of one hundred and twenty (120) residents were recruited to participate in this study; sixty (60) residents displayed BPSD (in accordance with PAI scores) while sixty (60) residents did not.

\section{Tools used in the study}

All of the measures are presented in the appendices. The following tools were used in this study:

\section{Mini-mental state examination (MMSE)}

The Mini-Mental State Examination (MMSE) is a commonly used, evidence-based scale which screens for cognitive impairment in a reliable and valid way. It consists of twelve (12) questions that assess orientation, short-term memory (retention and recall), and language. The maximum score on the Mini Mental State Exam is thirty (30); scores of twenty-three (23) or less indicate cognitive impairment [22] A score of nineteen (19) or less correlates with moderate to advanced $\mathrm{D} / \mathrm{NCD}[22]$.

\section{Pittsburg agitation inventory (PAI)}

The Pittsburg Agitation Inventory (PAI) provides quantification of the severity of disruptive behaviors within the four most problematic behaviors associated with agitation:

\section{Aberrant vocalization}

2. Motor agitation

3. Aggressiveness

4. Resistance to care

The reliability and validity of the PAI is well established [24]

\section{Luthra's Behavioral Assessment and Intervention Response (LuBAIR) Scale}

Detail on the structure and format of LuBAIR Scale has been described in the introduction section. LuBAIR Scale has been specifically developed for moderate to advanced D/NCD patients presenting with behaviors. Whereas, the severity of behaviors in community setting is often determined on the basis of care giver stress [25], there is limited guidance from literature in defining the severity of behaviors in care and institutional settings. For LuBAIR scale, severity is defined on the basis of individual behaviors response to interpersonal interventions (IPI). The severity ranges from not present (NP), sustained response to IPI (mild), non-sustained response to IPI (moderate) and no response to IPI (severe). LuBAIR does not capture the frequency and duration of individual behavioral symptoms in their respective categories. This is accomplished by transferring the twelve (12) defined behavioral categories (not individual symptoms) to a Dementia Observation Scale (DOS), also referred to as Q-30 minute scale. 
Citation: Luthra AS (2015) A New Behavioural Assessment Tool for Patients with Major Neurocognitve Disorders: Results of a Clinical Study. J Gerontol Geriatr Res 4: 260. doi:10.4172/2167-7182.1000260

Page 4 of 9

\section{Cohen-mansfield agitation inventory (CMAI)}

The Cohen-Mansfield Agitation Inventory (CMAI) is a twentynine (29) item caregiver rating questionnaire for the assessment of agitation in elderly persons. It includes descriptions of twenty-nine (29) agitated behaviors; each rated on a seven (7) point scale of frequency and intensity. Inter-rater correlation coefficients ranged between 0.88 and 0.92 [9]

\section{Behaviors- alzheimer disease (BEHAVE-AD)}

The Behavioral Pathology in Alzheimer's disease (BEHAVE-AD) rating scale was developed by Reisberg [14] to measure behavioral disturbances and psychiatric symptoms in patents with $\mathrm{D} / \mathrm{NCD}$. This scale consists of seven (7) behavioral categories (paranoid and delusional ideation, hallucinations, activity disturbances, aggressiveness, diurnal rhythm disturbances, affective disturbances, anxieties, and phobias) and consists of twenty-five (25) symptoms grouped into these categories. Each of these categories has to be scored independently [26]. Conducted a study to evaluate frequency weighted scores to the BEHAVE-AD and found intra-rater correlation coefficient in the range of 0.86 to 0.97 .

\section{Clinical utility survey (CUS)}

CUS was specifically developed for this study. CUS was intended to be completed by nurses who filled out all the study tools including LuBAIR scale for the study population. The primary purpose of CUS was to evaluate the usefulness of this scale in clinical practice by asking nurse participants the following questions (yes/no response):

(1) Does the LuBAIR take less time to complete than other scales?

(2) Does it collect more information than other scales?

(3) Does it help you understand behaviors in a clinically meaningful way?

\section{Participant recruitment}

Registered nurses (RN) identified residents who had a diagnosis of D/NCD on their respective units. These identified residents were divided into two (2) groups; with and without BPSD. The RA connected with the Power of Attorney (POA) or Substitute Decision Maker (SDM) for each of the identified residents to explain the details around the study, provide a letter of information, answer questions and obtain written consent from those interested in participating.

\section{Study design and methodology}

Seven (7) LTCFs, five (5) located in Hamilton, Ontario, Canada and two (2) in Burlington, Ontario, Canada agreed to participate in this study. Identified residents were screened in accordance with the inclusion criteria and a total of one hundred and twenty-five (120) residents were recruited to participate in the study.

All of the sixty (60) residents in the study group were deemed incapable of consenting to participate in the study. The POA or SDM for each of these residents in the study group provided informed consent in accordance with the Research Ethics Board guidelines. Of the sixty (60) residents in the control group, twenty-three (23) were deemed to be capable of consenting to participate in the study and signed their owned consent. Thirty-seven (37) of the residents in the control group were deemed to be incapable of consenting to participate in the study. Their POA or SDM consented to participate in the study.

RNs were asked to complete LuBAIR scale on the same residents on separate occasions, two (2) weeks apart, to calculate intra-rater reliability. A second group of RNs on each of the respective units were asked to complete LuBAIR scale on the same residents as the first RN group to calculate inter-rater reliability.

On the day RNs were asked to complete LuBAIR scale, they were also asked to complete the BEHAVE-AD and Cohen-Mansfield Agitation Inventory (CMAI) on the same set of residents in the study group. Once all of the above scales were completed on the residents in the study group, RN's were asked to complete the "clinical utility survey" (CUS).

Finally, four (4) geriatric specialists (two (2) geriatric psychiatry and two (2) geriatric medicine) were asked to review LuBAIR and provide feedback on i) the layout and title of the scale, ii) the ease of use of the scale, and iii) the content of the scale.

The estimated time for the completion of the study was twelve (12) months. The study began in January 2009 and continued until September 2011.

\section{Definition of End Points}

Data collection was deemed to be completed when the following reliability, validity, and additional endpoints were reached.

\section{Reliability endpoints}

- Correlations (agreement) between severity scores for each category of behaviors on LuBAIR scale collected at different points in time and by the same RN (intra-rater reliability).

- Correlations (agreement) between severity scores for each category of behaviors on LuBAIR scale collected at the same point in time by two different RNs (inter-rater reliability).

\section{Validity endpoints}

- Correlations between LuBAIR scale scores and scores on the BEHAVE-AD and CMAI.

- The proportion of residents who are correctly classified as having BPSD.

- The proportion of residents who do not have BPSD, who are classified as having BPSD.

- Face validity established by the four (4) geriatric specialists.

\section{Additional endpoints}

- Percentage of RNs who indicated that LuBAIR scale takes less time to complete than other scales.

- Percentage of RNs who indicated that LuBAIR scale collects more information than other scales.

- Percentage of RNs who indicated that LuBAIR scale helps them to understand behaviors in a clinically meaningful way.

\section{Results}

Screening results, reliability, and validity results of LuBAIR scale will be discussed in this section.

\section{Screening results}

The sample size calculation was done using the standard parameters;

- Population size: In the year the study was initiated (2009), there were 150,000 persons with dementia in Ontario [27],

- Confidence Interval: Margin of error was kept at 5\%.

- Confidence Level: This was chosen to be at $90 \%$. 


\begin{tabular}{|c|c|c|c|c|c|}
\hline Subject \# & Gender & Age & MMSE & PAS & Clock \\
\hline 1 & $\mathrm{~F}$ & 81 & 16 & 3 & $x$ \\
\hline 2 & $\mathrm{~F}$ & 85 & 9 & 5 & $x$ \\
\hline 3 & $\mathrm{~F}$ & 87 & 10 & 6 & $x$ \\
\hline 4 & $\mathrm{~F}$ & 87 & 14 & 6 & $x$ \\
\hline 5 & $\mathrm{~F}$ & 85 & 17 & 4 & $x$ \\
\hline 6 & $\mathrm{~F}$ & 79 & 17 & 3 & $Y$ \\
\hline 7 & $\mathrm{~F}$ & 94 & 5 & 7 & $x$ \\
\hline 8 & $\mathrm{M}$ & 80 & 6 & 3 & $X$ \\
\hline 9 & M & 81 & 5 & 5 & $\mathrm{x}$ \\
\hline 10 & $\mathrm{~F}$ & 88 & 1 & 8 & $x$ \\
\hline 11 & $M$ & 93 & 0 & 5 & $x$ \\
\hline 12 & $\mathrm{~F}$ & 82 & 11 & 3 & $x$ \\
\hline 13 & $\mathrm{~F}$ & 92 & 0 & 3 & $x$ \\
\hline 14 & $M$ & 88 & 6 & 5 & $\mathrm{x}$ \\
\hline 15 & $\mathrm{~F}$ & 81 & 19 & 8 & $\mathrm{Y}$ \\
\hline 16 & $M$ & 83 & 0 & 4 & $x$ \\
\hline 17 & $\mathrm{M}$ & 77 & 4 & 5 & $x$ \\
\hline 18 & $\mathrm{~F}$ & 94 & 0 & 8 & $x$ \\
\hline 19 & $\mathrm{~F}$ & 88 & 4 & 7 & $x$ \\
\hline 20 & $M$ & 89 & 11 & 11 & $x$ \\
\hline 21 & $\mathrm{M}$ & 93 & 6 & 4 & $x$ \\
\hline 22 & $\mathrm{M}$ & 84 & 19 & 8 & $x$ \\
\hline 23 & M & 91 & 14 & 12 & $x$ \\
\hline 24 & $\mathrm{~F}$ & 97 & 0 & 9 & $x$ \\
\hline 25 & $\mathrm{~F}$ & 77 & 0 & 6 & $x$ \\
\hline 26 & $\mathrm{~F}$ & 79 & 0 & 9 & $\mathrm{x}$ \\
\hline 27 & $M$ & 74 & 12 & 5 & $x$ \\
\hline 28 & $\mathrm{~F}$ & 80 & 0 & 7 & $x$ \\
\hline 29 & $\mathrm{~F}$ & 76 & 5 & 10 & $x$ \\
\hline 30 & $\mathrm{~F}$ & 79 & 0 & 5 & $x$ \\
\hline 31 & $\mathrm{~F}$ & 82 & 1 & 5 & $X$ \\
\hline 32 & $\mathrm{~F}$ & 81 & 0 & 5 & $X$ \\
\hline 33 & $\mathrm{~F}$ & 87 & 4 & 3 & $X$ \\
\hline 34 & $\mathrm{M}$ & 78 & 17 & 11 & $x$ \\
\hline 35 & $M$ & 97 & 8 & 7 & $x$ \\
\hline 36 & $\mathrm{~F}$ & 98 & 9 & 10 & $X$ \\
\hline 37 & $\mathrm{~F}$ & 78 & 9 & 4 & $\mathrm{x}$ \\
\hline 38 & $\mathrm{~F}$ & 83 & 7 & 3 & $X$ \\
\hline 39 & $\mathrm{M}$ & 84 & 4 & 4 & $x$ \\
\hline 40 & $\mathrm{~F}$ & 100 & 0 & 4 & $\mathrm{x}$ \\
\hline 41 & $\mathrm{~F}$ & 81 & 11 & 6 & $Y$ \\
\hline 42 & $\mathrm{~F}$ & 93 & 2 & 13 & $x$ \\
\hline 43 & $\mathrm{~F}$ & 88 & 19 & 11 & $x$ \\
\hline 44 & $\mathrm{M}$ & 70 & 0 & 8 & $X$ \\
\hline 45 & $M$ & 67 & 0 & 4 & $X$ \\
\hline 46 & $\mathrm{~F}$ & 86 & 0 & 7 & $X$ \\
\hline 47 & $\mathrm{M}$ & 86 & 18 & 5 & $x$ \\
\hline 48 & $\mathrm{~F}$ & 89 & 16 & 5 & $x$ \\
\hline 49 & $\mathrm{~F}$ & 89 & 17 & 3 & $x$ \\
\hline 50 & $\mathrm{M}$ & 82 & 7 & 12 & $x$ \\
\hline 51 & $\mathrm{~F}$ & 86 & 3 & 9 & $x$ \\
\hline 52 & $\mathrm{~F}$ & 81 & 17 & 6 & $\mathrm{x}$ \\
\hline 53 & $\mathrm{M}$ & 84 & 2 & 9 & $x$ \\
\hline 54 & $\mathrm{~F}$ & 80 & 9 & 7 & $X$ \\
\hline 55 & M & 78 & 12 & 6 & $x$ \\
\hline 56 & $\mathrm{~F}$ & 77 & 8 & 9 & $x$ \\
\hline 57 & $\mathrm{M}$ & 81 & 14 & 5 & $x$ \\
\hline 58 & $M$ & 80 & 8 & 5 & $X$ \\
\hline 59 & $\mathrm{~F}$ & 83 & 14 & 6 & $x$ \\
\hline 60 & $\mathrm{~F}$ & 79 & 5 & 8 & $x$ \\
\hline
\end{tabular}

Table 1: Score on MMSE and PAS for study group.
[28] program was used to calculate the sample size at two hundred and seventy (270). However, based upon the logistical challenges experienced in conducting the study, it would have taken another three (3) years to complete the study. Therefore, an executive decision was made to raise the margin of error to $7.5 \%$. This resulted in reducing the sample size to one hundred and twenty (120); the final number chosen for the study.

One hundred and twenty (120) residents were included in the study and in accordance with inclusion and exclusion criteria. See Table 1 for details of MMSE and PAS scores for sixty (60) residents in the study group and Table 2 for MMSE and PAS scores for sixty (60) in the control group. See Table 3 for the average MMSE and PAS scores for the study group.

\begin{tabular}{|c|c|c|c|c|c|}
\hline Subject \# & Gender & Age & MMSE & PAS & Clock \\
\hline 1 & $\mathrm{~F}$ & 81 & 21 & 2 & $x$ \\
\hline 2 & $\mathrm{~F}$ & 84 & 11 & 2 & $\mathrm{x}$ \\
\hline 3 & $\mathrm{~F}$ & 85 & 19 & 1 & $x$ \\
\hline 4 & $\mathrm{~F}$ & 89 & 22 & 0 & $\mathrm{Y}$ \\
\hline 5 & $M$ & 72 & 15 & 2 & $x$ \\
\hline 6 & $M$ & 89 & 12 & 2 & $X$ \\
\hline 7 & $M$ & 89 & 15 & 2 & $x$ \\
\hline 8 & $\mathrm{~F}$ & 89 & 14 & 2 & $\mathrm{x}$ \\
\hline 9 & M & 92 & 11 & 0 & $x$ \\
\hline 10 & $\mathrm{~F}$ & 88 & 16 & 0 & $x$ \\
\hline 11 & $\mathrm{~F}$ & 79 & 17 & 0 & $x$ \\
\hline 12 & $M$ & 88 & 12 & 0 & $x$ \\
\hline 13 & M & 98 & 19 & 2 & $\mathrm{x}$ \\
\hline 14 & $\mathrm{~F}$ & 80 & 20 & 1 & $\mathrm{x}$ \\
\hline 15 & $\mathrm{~F}$ & 89 & 24 & 2 & $x$ \\
\hline 16 & $\mathrm{~F}$ & 79 & 17 & 0 & $x$ \\
\hline 17 & $M$ & 89 & 22 & 2 & $x$ \\
\hline 18 & $\mathrm{~F}$ & 81 & 20 & 2 & $x$ \\
\hline 19 & $\mathrm{~F}$ & 84 & 19 & 2 & $x$ \\
\hline 20 & $\mathrm{~F}$ & 85 & 19 & 1 & $\mathrm{x}$ \\
\hline 21 & $\mathrm{~F}$ & 89 & 16 & 0 & $x$ \\
\hline 22 & $\mathrm{~F}$ & 81 & 16 & 1 & $x$ \\
\hline 23 & $M$ & 79 & 18 & 0 & $x$ \\
\hline 24 & $M$ & 85 & 23 & 2 & $x$ \\
\hline 25 & $M$ & 83 & 21 & 1 & $X$ \\
\hline 26 & $\mathrm{~F}$ & 81 & 20 & 1 & $x$ \\
\hline 27 & $\mathrm{~F}$ & 87 & 21 & 2 & $x$ \\
\hline 28 & $\mathrm{~F}$ & 89 & 18 & 0 & $x$ \\
\hline 29 & $M$ & 81 & 19 & 2 & $x$ \\
\hline 30 & $\mathrm{~F}$ & 82 & 16 & 1 & $x$ \\
\hline 31 & $\mathrm{~F}$ & 80 & 20 & 0 & $x$ \\
\hline 32 & $\mathrm{~F}$ & 81 & 19 & 1 & $x$ \\
\hline 33 & M & 88 & 17 & 0 & $x$ \\
\hline 34 & $\mathrm{~F}$ & 89 & 15 & 1 & $x$ \\
\hline 35 & $M$ & 85 & 18 & 2 & $x$ \\
\hline 36 & $\mathrm{~F}$ & 79 & 15 & 1 & $x$ \\
\hline 37 & $\mathrm{~F}$ & 81 & 19 & 1 & $x$ \\
\hline 38 & $M$ & 88 & 16 & 2 & $x$ \\
\hline 39 & $\mathrm{~F}$ & 82 & 17 & 0 & $x$ \\
\hline 40 & $\mathrm{~F}$ & 83 & 18 & 0 & $x$ \\
\hline 41 & $M$ & 78 & 19 & 1 & $x$ \\
\hline 42 & $\mathrm{~F}$ & 89 & 15 & 1 & $x$ \\
\hline 43 & $\mathrm{~F}$ & 80 & 14 & 0 & $x$ \\
\hline 44 & $\mathrm{~F}$ & 78 & 19 & 1 & $x$ \\
\hline 45 & $\mathrm{~F}$ & 82 & 17 & 2 & $x$ \\
\hline 46 & $\mathrm{~F}$ & 86 & 18 & 1 & $x$ \\
\hline
\end{tabular}




\begin{tabular}{|l|l|l|l|l|l|}
\hline 47 & M & 79 & 13 & 1 & $\mathrm{X}$ \\
\hline 48 & $\mathrm{M}$ & 78 & 16 & 0 & $\mathrm{X}$ \\
\hline 49 & $\mathrm{~F}$ & 87 & 12 & 0 & $\mathrm{X}$ \\
\hline 50 & $\mathrm{~F}$ & 89 & 13 & 1 & $\mathrm{X}$ \\
\hline 51 & $\mathrm{~F}$ & 81 & 15 & 0 & $\mathrm{X}$ \\
\hline 52 & $\mathrm{M}$ & 83 & 17 & 1 & $\mathrm{X}$ \\
\hline 53 & $\mathrm{M}$ & 89 & 19 & 1 & $\mathrm{X}$ \\
\hline 54 & $\mathrm{~F}$ & 79 & 16 & 0 & $\mathrm{X}$ \\
\hline 55 & $\mathrm{~F}$ & 87 & 17 & 1 & $\mathrm{X}$ \\
\hline 56 & $\mathrm{~F}$ & 85 & 16 & 1 & $\mathrm{X}$ \\
\hline 57 & $\mathrm{M}$ & 82 & 14 & 1 & $\mathrm{X}$ \\
\hline 58 & $\mathrm{~F}$ & 83 & 13 & 1 & $\mathrm{X}$ \\
\hline 59 & $\mathrm{~F}$ & 78 & 18 & 1 & $\mathrm{X}$ \\
\hline 60 & $\mathrm{~F}$ & 81 & 19 & 0 & $\mathrm{X}$ \\
\hline
\end{tabular}

Table 2: Score on MMSE and PAS for control group.

\begin{tabular}{|c|c|c|c|}
\hline Scale & Average score & Lowest score & Highest score \\
\hline MMSE & 7.67 & 0 & 22 \\
\hline PAS & 6.38 & 3 & 13 \\
\hline
\end{tabular}

Screening cut-off for MMSE was set at 23 or below. Screening cut-off for PAS was set at 3 or above $\mathrm{N}=60$

Table 3: Average Score on MMSE and PAI in behavior group.

\begin{tabular}{|c|c|c|}
\hline $\begin{array}{c}\text { Clinical Categories } \\
\text { for LuBAIR }\end{array}$ & Intraclass Correlations (95\% & value) \\
\hline Disorganized & $0.269(0.003-0.500)$ & $0.024^{*}$ \\
\hline Misidentification & $0.198(-0.073-0.441)$ & 0.075 \\
\hline Motor & $0.310(0.047-0.533)$ & $<0.0001^{*}$ \\
\hline Goal Directed & $0.476(0.240-0.659)$ & $<0.0001^{*}$ \\
\hline Vocally Disruptive & $0.472(0.235-0.657)$ & $<0.0001^{*}$ \\
\hline Emotional & $0.642(0.453-0.776)$ & $<0.0001^{*}$ \\
\hline Importuning & $0.430(0.184-0.625)$ & $0.001^{*}$ \\
\hline Fretful & $0.026(-0.242-0.291)$ & 0.425 \\
\hline Apathy & $0.271(0.004-0.501)$ & $0.023^{*}$ \\
\hline Oppositional & $0.445(0.203-0.637)$ & $<0.0001^{*}$ \\
\hline Physically Aggressive & $0.638(0.448-0.773)$ & $<0.0001^{*}$ \\
\hline Sexual & $0.436(0.191-0.630)$ & $0 .<0.0001^{*}$ \\
\hline
\end{tabular}

One-way random effects model where people effects are random. $\alpha=0.05$.

* Significant findings $(p<\alpha=0.05)$

Table 4: Inter-rater reliability coefficients.

\begin{tabular}{|c|c|c|}
\hline $\begin{array}{c}\text { Clinical Categories } \\
\text { for LuBAIR }\end{array}$ & Intraclass Correlations (95\% & \\
\hline Disorganized & $0.502(0.206-0.715)$ & $0.001^{*}$ \\
\hline Misidentification & $-0.024(-0.352-0.311)$ & 0.554 \\
\hline Motor & $0.498(0.200-0.712)$ & $0.001^{*}$ \\
\hline Goal Directed & $0.481(0.178-0.701)$ & $0.002^{*}$ \\
\hline Vocally Disruptive & $0.595(0.329-0.774)$ & $<0.0001^{*}$ \\
\hline Emotional & $0.373(0.048-0.627)$ & $0.013^{*}$ \\
\hline Importuning & $0.472(0.167-0.695)$ & $0.002^{*}$ \\
\hline Fretful & $-0.035(-0.361-0.301)$ & 0.578 \\
\hline Apathy & $0.171(-0.169-0.476)$ & 0.160 \\
\hline Oppositional & $0.316(-0.016-0.587)$ & $0.031^{*}$ \\
\hline Physically Aggressive & $0.641(0.393-0.802)$ & $<0.0001^{*}$ \\
\hline Sexual & $-0.041(-0.367-0.295)$ & 0.592 \\
\hline
\end{tabular}

One-way random effects model where people effects are random. $\alpha=0.05$.

* Significant findings $(p<\alpha=0.05)$

Table 5: Intra-rater reliability coefficients.

\section{Inter-rater reliability}

Inter-rater reliability was calculated using SPSS Statistical Software. Please refer to Table 4 for detailed results of the inter-rater correlation coefficient for the individual behavioral categories on LuBAIR scale. Ten (10) out of the twelve (12) categories showed statistically significant correlations, with the strongest correlations for the "emotional" and "physically aggressive" behavior categories. The categories that did not show a statistically significant inter-rater reliability were the "misidentification" and "fretful/trepidated" behavior categories.

\section{Intra-rater reliability}

Intra-rater reliability was calculated using SPSS Statistical Software. Please refer to Table 5 for detailed results for intra-rater correlation coefficient for individual behavioral categories on LuBAIR scale. Eight (8) out of the twelve (12) categories showed statistically significant correlations, with the strongest correlations for the "physically aggressive" and "vocal" behavior categories. The behavior categories that did not show a statistically significant inter-rater reliability were the "misidentification", "fretful/trepidated", "apathy" and "sexual" categories.

\section{Content and criterion validity}

Total scores from LuBAIR scale, CMAI and BEHAVE-AD scales were used to calculate inter-scale correlation coefficients using Pearson's 2-tailed test. Table 6 describes detailed results for the first rater who filled out all three scales.

As seen in Table 6, all inter-scale correlation coefficients were found to be significant. Table 7 describes detailed results for the second rater who filled out all three scales on each resident.

Table 8 describes inter rater correlation coefficient for the total scores calculated for LuBAIR, CMAI and BEHAVE-AD for each of the residents in the study group.

As seen in Table 6, all inter-scale correlation coefficients were found to be significant.

\section{Face validity}

Four (4) geriatric specialists provided feedback on the following:

\section{Layout of the scale including title}

This was found to be acceptable by all four (4) geriatric specialists. Two (2) of the four (4) geriatric specialists would have liked to see a "frequency" measure added to scale, much like the CMAI. Most of the criticism was directed towards the initial titles of the scale. The two (2) titles which were initially chosen were Luthra's Behavioral Inventory in Dementia (L-BID) and Luthra's Inventory of Behaviors in Dementia (LIBID). Both of the acronyms were reported to be effortful in the daily use in clinical practice. It was suggested that there be search for a 'softer' name for use in clinical practice. Hence, the final name chosen was LuBAIR scale, amongst other names, after informal feedback from front line staff.

\section{Ease of use}

All four (4) of the specialized geriatric specialist did not comment

\begin{tabular}{|c|c|c|c|c|}
\hline & LuBAIR & CMAI & BEHAVE-AD & \\
\hline \multirow{2}{*}{ LuBAIR } & 1 & $0.764^{*}$ & $0.782^{*}$ & Pearson Correlation \\
\hline & - & $<0.0001$ & $<0.0001$ & Sig. (2-tailed) \\
\hline \multirow{2}{*}{ CMAI } & $0.764^{*}$ & 1 & $0.678^{*}$ & Pearson Correlation \\
\hline & $<0.0001$ & & $<.0001$ & Sig. (2-tailed) \\
\hline \multirow{2}{*}{ BEHAVE-AD } & $0.782^{*}$ & $0.678^{*}$ & 1 & Pearson Correlation \\
\hline & $<0.0001$ & $<0.0001$ & - & Sig. (2-tailed) \\
\hline
\end{tabular}

* Correlation is significant at or below the 0.01 level (2-tailed). $\mathrm{N}=60$. Table 6: Scale Cross-validation with Total Scores-Rater 1 


\begin{tabular}{|c|c|c|c|c|}
\hline & LuBAIR & CMAI & BEHAVE-AD & \\
\hline \multirow{2}{*}{ LuBAIR } & 1 & $0.643^{*}$ & $0.575^{\star}$ & Pearson Correlation \\
\hline & - & $<0.0001$ & $<0.0001$ & Sig. (2-tailed) \\
\hline \multirow{2}{*}{ CMAI } & $0.643^{*}$ & 1 & $0.496^{*}$ & Pearson Correlation \\
\hline & $<0.0001$ & - & 0.001 & Sig. (2-tailed) \\
\hline \multirow{2}{*}{ BEHAVE-AD } & $0.575^{*}$ & $0.496^{*}$ & 1 & Pearson Correlation \\
\hline & 0.000 & 0.001 & - & Sig. (2-tailed) \\
\hline
\end{tabular}

* Correlation is significant at or below the 0.01 level (2-tailed). $\mathrm{N}=60$

Table 7: Scale Cross-validation with Total Scores-Rater 2

\begin{tabular}{|c|c|c|c|c|}
\hline & & \multicolumn{3}{|c|}{ RATER 2} \\
\hline & & LuBAIR & CMAI & BEHAVE-AD \\
\hline \multirow{3}{*}{ 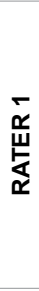 } & LuBAIR & $\begin{array}{c}0.320^{*} \\
p=0.020 \\
N_{R 1}=53, N_{R 2}=53\end{array}$ & - & - \\
\hline & CMAI & 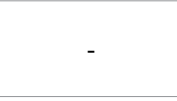 & $\begin{array}{c}0.394^{*} \\
p=0.008 \\
N_{R 1}=53, N_{R 2}=44\end{array}$ & - \\
\hline & BEHAVE-AD & - & - & $\begin{array}{c}0.386^{*} \\
p=0.010 \\
N_{R 1}=53, N_{R 2}=44\end{array}$ \\
\hline
\end{tabular}

* Correlation is significant at or below the 0.05 level (2-tailed). $\mathrm{N}_{\mathrm{R} 1}=$ Participants assessed by Rater $1 ; \mathrm{N}_{\mathrm{R} 2}=$ Participants assessed by Rater 2 .

Table 8: Rater cross-validation with total scores.

one way or the other as to the ease of use of the scale. All were of the opinion the scales would be initially filled for them by the trained registered staff. They would become involved at the next stage of reviewing the clinical notes to ensure the accuracy of the interpretation of the meaning of the identified symptoms in the clinical records.

\section{Content of the scale}

All the geriatric specialists were satisfied with the exhaustive nature of the behavioral symptoms collected in the scale.

\section{Clinical utility}

RNs were asked to answer three (3) survey questions after completion of the LuBAIR scale on each study participant. Data are available for forty-five (45) out of fifty-eight (58) (78\%) assessments with the LuBAIR scale. The results of this survey are as follows:

Question 1: Does the LuBAIR scale take less time to complete than other scales? YES: $24 \%$. NO: $76 \%$.

Question 2: Does the LuBAIR scale collect more information than other scales? YES: $82 \%$. NO: $18 \%$.

Question 3: Does the LuBAIR scale help you understand behaviors in a clinically meaningful way? YES: $98 \%$. NO: $2 \%$.

\section{Discussion}

The study did take longer than the scheduled allotted time to complete. The primary reason for delay in completion of the study was the lack of time availability by the registered staff to make themselves available to the research assistant (RA). Since the staffing models are determined by financial constraints, one (1) RN is often responsible for overseeing more than one (1) clinical unit in LTCF. Fatigue at the end of the shift, emergencies and other last minute responsibilities often resulted in rescheduling of the appointments with the RA. Prolongation of the study resulted in the initial funding running out and the first RA moved on due to admission to a professional school. Application for new funding and training of a new RA resulted in obvious delays in completion of the study. A significant part of the author's clinical work is in LTCF and the study duration and funding was based on the author's experience of the operations of LTCF. Clearly the author grossly underestimated the overall work load carried by the registered staff on their respective shifts. It gave the author a new found respect for the staff working in LTCF.

The delay in submission of the manuscript for publication was intentional. Frequently raised questions at clinical presentations of the results of the study were centered on the rationale for proposing new terminology and classification for behaviors in moderate to advanced $\mathrm{D} / \mathrm{NCD}$. Likewise, explanations were sought for the rationale used to attribute the proposed meaning for each behavioral category. The need for a compendium which outlined all the theoretical constructs used to create this new terminology and behavioral classification became rather apparent. This focus led to the writing of the book titled; The Meaning of Behaviors in Dementia/Neurocognitive Disorders; New Terminology, Classification and Behavioral Intervention. This book was published by Common Ground Press (Journal of Aging and Society) in September 2014. It is available on Amazon.com. With the book in place, submission of the manuscript of the study was the next logical step.

Inter-rater reliability was well within acceptable range for ten (10) of the twelve (12) behavioral categories when compared to CMAI and BEHAVE-AD. The two (2) categories which failed to reach acceptable levels of reliability were 'misidentification' and 'fretful/trepidated' behaviors. Intra-rater reliability was found to be reaching statistical significance in eight (8) of the twelve (12) behavioral categories. The "misidentification", "fretful/trepidated", "apathy", and "sexual" categories were the four (4) behavioral categories which failed to reach statistical significance.

Failure to reach statistical significance in the three (3) of the four (4) identified categories (Misidentification, Fretful/Trepidated and Apathy) can be explained on the basis of lack of familiarity with the newly appointed terminology. Front line staff were extremely familiar with terms like paranoia, 'delusions' and 'hallucinations' but not at all familiar with terms like Capgras and Fregoli syndrome, which are used to describe the "Misidentification" category. Once the explanation was provided to the staff using concrete examples, they were readily able to identify these Misidentification symptoms in their daily interactions with the residents. In the early stages of $\mathrm{D} / \mathrm{NCD}$, staff members are able to conduct a reliable and valid clinical interview to determine the fixedness and falseness of the beliefs verbalized by the resident. The same approach would be needed to clarify for the presence of perceptions in the absence of stimulus. However, in residents with moderate to advanced D/NCD, when such a clinical interview is not possible, reliability and validity of clinical symptoms of delusions and hallucination cannot be established. Likewise, behavioral symptoms represented under the 'Fretful/Trepidated' category are frequently alluded to as 'anxiety' symptoms. Absence of the term "anxiety" under the definition of "Fretful/Trepidated" may have resulted in staff struggling to find a home of these symptoms.

In clinical practice, "Apathy" behaviors are more commonly referred to as "depressed" behaviors. The latter term is conspicuously absent from this scale and this was done on purpose. Psychology literature has scientific definitions for terms 'emotions' and 'mood' and they are not interchangeable [20]. Patients with moderate to advanced D/NCD are not expected to track, register and recall their emotional states over duration of several days to weeks. The latter is a pre-requisite to qualify for an emotional state as 'mood' [20]. Likewise, the degree of cognitive impairment in the same patient population will impair their ability to track, register and recall changes in interest levels and/or ability to 
Citation: Luthra AS (2015) A New Behavioural Assessment Tool for Patients with Major Neurocognitve Disorders: Results of a Clinical Study. J Gerontol Geriatr Res 4: 260. doi:10.4172/2167-7182.1000260

Page 8 of 9

experience pleasure over days and weeks. Changes in the latter two symptoms are mandatory criteria to qualify for a diagnosis of Major Depressive Episode in accordance with Diagnostic and Statistical Manual (DSM-V) [10]. Therefore, observing and tracking changes in emotional states in this patient population is a much more reliable and valid measure.

Personal and societal value systems and the environment play a significant role in labeling behaviors as either "sexual" or not [16]. Behaviors labelled as 'sexual' on day one of their occurrence may not qualify as being sexual in nature once all the facts are reviewed [16]. A combination of the above factors likely accounted for variability in the labelling of 'sexual' behaviors in this research study.

Content and criteria validity was established using inter-scale reliability from the LuBAIR scale, CMAI and BEHAVE-AD scales filled out by $\mathrm{RN}$ group 1 and $\mathrm{RN}$ group 2 . All of these results reached statistically significant proportions. It is therefore reasonable to stipulate LuBAIR scale captures behaviors which are meant to be captured and no residents were labeled as having BPSD who did not have such behaviors.

The results of the Clinical Utility Survey (CUS) ought to be interpreted in accordance with the raised margin of error in sample size calculation. CUS was designed to gather qualitative data around LuBAIR scale's applicability in a clinical setting. It was surprising the results for the first question in CUS did not support stated hypothesis (LuBAIR scale would be less labor intensive). Further discussions with clinical staff revealed the newness of the scale, its different layout, and the need to have a comprehensive conceptual understanding of individual behavioral categories scale as a pre-requisite to using it in an optimal manner. The RNs did comment that once this level of understanding was accomplished, with repetition of learning sessions, perhaps it would lend itself to faster data collection. The RNs did support the hypothesis that LuBAIR scale was able to collect much more information in comparison to the other two scales. Furthermore, the RNs also supported the hypothesis that LuBAIR scale assisted them in gathering information under clinically meaningful behavioral categories, thereby helping them understand the 'purpose' or 'meaning' of behaviors.

\section{Conclusions}

LuBAIR scale has comparable inter- and intra-rater reliability in comparison to existing behavioral scales CMAI and BEHAVE-AD. Likewise, LuBAIR scale also has comparable content and criteria validity in comparison to these existing scales. Its advantage appears to be in the realm of collecting more data than the existing scales and allowing for data to be put under clinically meaningful categories in order to help with understanding the 'meaning' of observed behaviors.

No conflict of interest presented. Seed money to the amount of $\$ 5000.00$ was obtained from Regional Geriatric Program (central) (RGPC), Ontario, Canada. would like thank Davis Jewell, Executive Director RGPc, for his support for the study. Funding to the amount of $\$ 3000.00$ was obtained from the Community Division, Homewood Health Center. Balance of the funding for the study to the amount of $\$ 2000.00$ was incurred by the author.

\section{Acknowledgments}

I would like to thank Deanna Porter and Heather Foster, Murray Alzheimer's Research and Education Program (MAREP) research scholars, 2009 and 2010, respectively, in conducting the clinical study. I would like to thank Heather Foste for writing the initial drafts of the study and performing all the statistical analysis on the data. I would like to thank Trevor Semplois (MAREP research scholar, 2012) for continued assistance on this and several other projects including the writing of the text book and finalizing of this manuscript. Finally, I would like to thank Yarima Gonzalez for assisting in the final formatting of the manuscript and making it submission ready.

\section{References}

1. Liperoti R, Pedone C, Corsonello A (2008) Antipsychotics for the treatment of behavioral and psychological symptoms of dementia (BPSD). Curr Neuropharmacol 6: 117-124.

2. Lockhart J, Lestage PJ (2003) "Cognition Enhancing Or Neuroprotective Compounds for the Treatment of Cognitive Disorders: Why? when? which?" Experimental Gerontology 38: 119-128.

3. Margallo-Lana M, Swann A, O'Brien J, Fairbairn A, Reichelt K, et al (2001) "Prevalence and Pharmacological Management of Behavioural and Psychological Symptoms Amongst Dementia Sufferers Living in Care Environments." Int J Geriatr Psychiatry 16: 39-44.

4. Douglas S, James I, Ballard C (2004) Non-pharmacological interventions in dementia. Advances in Psychiatric Treatment $10: 171-177$

5. Takeda M, Tanaka T, Okochi M, Kazui H (2012) "Non-pharmacological Intervention for Dementia Patients." Psychiatry and Clinical Neurosci 66: 1-7.

6. Conn D, Thorpe L (2007) Assessment of behavioural and psychological symptoms associated with dementia. Can J Neurol Sci 34 Suppl 1: S67-71.

7. http://whqlibdoc.who.int/publications/2012/9789241564458_eng.pdf

8. Cohen-Mansfield J, Billig N (1986) Agitated behaviors in the elderly. I. A conceptual review. J Am Geriatr Soc 34: 711-721.

9. Cohen-Mansfield $\mathrm{J}$ (1995) Assessment of disruptive behavior/agitation in the elderly: function, methods, and difficulties. J Geriatr Psychiatry Neurol 8: 52-60.

10. American Psychiatric Association (2013) Diagnostic and statistical manual of mental disorders. (5thedn) American Psychiatric Association, Arlington.

11. Alexopoulos GS, Abrams RC, Young RC, Shamoian CA (1988) Cornell Scale for Depression in Dementia. Biol Psychiatry 23: 271-284.

12. Sunderland T1, Minichiello M (1996) Dementia Mood Assessment Scale. Int Psychogeriatr 8 Suppl 3: 329-331.

13. de Medeiros K, Robert P, Gauthier S, Stella F, A. Politis, et al. (2010) The Neuropsychiatric Inventory-Clinician Rating Scale (NPI-C): Reliability and Validity of a Revised Assessment of Neuropsychiatric Symptoms in Dementia Int Psychogeriatr 22: 984-994.

14. Reisberg B, Auer SR, Monteiro IM (1996) Behavioral pathology in Alzheimer's disease (BEHAVE-AD) rating scale.Int Psychogeriatr 8 Suppl 3: 301-308.

15. Inouye SK, Bogardus ST Jr, Charpentier PA, Leo-Summers L, Acampora D (1999) A multicomponent intervention to prevent delirium in hospitalized older patients. N Engl J Med 340: 669-676.

16. Sherry LD, Wiersma E and Loiselle L (2012) Pathologizing behavior: Meanings of behaviors in dementia care. Journal of Aging Studies 26: 162-173.

17. Skovdahl K1, Kihlgren AL, Kihlgren M (2003) Different attitudes when handling aggressive behaviour in dementia--narratives from two caregiver groups. Aging Ment Health 7: 277-286.

18. Morandi A, McCurley J, Vasilevskis EE, Fick DM, Bellelli G, et al. (2012) Tools to Detect Delirium Superimposed on Dementia: A Systematic Review.Journal of the American Geriatrics Society 60: 2005-2013.

19. Spector A, Orrell M (2010) Using a biopsychosocial model of dementia as a tool to guide clinical practice. Int Psychogeriatr 22: 957-965.

20. Luthra AS (2014) The Meaning of Behaviors in Dementia/Neurocognitive Disorders: New Terminology, Classification and Behavioral Management (1stedn), Common Ground Publishing Champaign, IL, USA.

21. Davis LL, Buckwalter K, Burgio LD (1997) Measuring problem behaviors in dementia: developing a methodological agenda. ANS Adv Nurs Sci 20: 40-55.

22. Folstein MF, Folstein SE, McHugh PR (1975) Mini-mental state: A practica method for grading the cognitive state of patients for the clinician.J Psychiatr Res 12: 189-198.

23. Hort J, O'Brien JT, Gainotti G, Pirttila T, Popescu BO, et al. (2010) EFNS guidelines for the diagnosis and management of Alzheimer's disease. Eur $\mathrm{J}$ Neurol 17: 1236-1248. 
Citation: Luthra AS (2015) A New Behavioural Assessment Tool for Patients with Major Neurocognitve Disorders: Results of a Clinical Study. J Gerontol Geriatr Res 4: 260. doi:10.4172/2167-7182.1000260

24. Rosen J, Burgio L, Kollar M, Cain M, Allison M, et al. (1994) The Pittsburgh Agitation Scale: A user-friendly instrument for rating agitation in dementia patients. Am J Geriatr Psychiatry 2: 52-59.

25. Sheehan B (2012) Assessment scales in dementia.Ther Adv Neurol Disord 5 349-358.

26. Monteiro IM, Boksay I, Auer SR, Torossian C, Ferris SH, et al. (2001) Addition of a frequency-weighted score to the Behavioral Pathology in Alzheimer's Disease Rating Scale: the BEHAVE-AD-FW: methodology and reliability.Eur Psychiatry 16 Suppl 1: 5s-24s.

27. Alzheimer's Society of Canada (2009) Rising Tide: The Impact of Dementia on Canadian Society. Alzheimer Society.

28. www.surveymonkey.com 